\title{
GRADED SUBALGEBRAS OF THE LIE ALGEBRA OF A SMOOTH MANIFOLD
}

\author{
KARL DE PAEPE
}

(Received 16 June 2000 and in revised form 16 December 2000)

\begin{abstract}
We construct and study finitely generated graded subalgebras of the Lie algebra of a smooth manifold.
\end{abstract}

2000 Mathematics Subject Classification. 17B66, 17B70.

1. Introduction. Let $M$ be a smooth manifold of dimension $n>1$. Embed $M$ in $\mathbb{R}^{m}$ for some $m>n$ so that the resulting point set forms an analytic manifold with no boundary. That this is possible follows from Whitney [1]. Let $\mathscr{Y}(M)$ be the Lie algebra of smooth vector fields on $M$ that smoothly go to zero at infinity if $M$ is not compact.

We will look at two types of finitely generated graded subalgebras of $\mathscr{S}(M)$. In one type we look at the Lie algebra generated by $n$ analytic vector fields that span the tangent space at some point of $M$ and such that no subset generates a finite dimensional Lie algebra. It is shown that this is a graded Lie algebra and that the graded subalgebras of two manifolds are isomorphic if and only if the manifolds are diffeomorphic. The other type is constructed from an atlas of the manifold satisfying some conditions.

2. Graded subalgebras with $\operatorname{dim} M$ number of generators. Without loss of generality, we set $n=2$ in this section. Choose two analytic vector fields $X_{j} \in \mathscr{S}(M)$ that span the tangent space for some point of $M$ and such that $m$ is infinite dimensional where $\mathfrak{m}=\mathfrak{m}\left(X_{1}, X_{2}\right)=\left\langle X_{1}, X_{2}\right\rangle$ is the Lie algebra generated by the $X_{j}$. We have $\mathfrak{m}=\sum_{k=1}^{\infty} \mathfrak{m}_{k}\left(X_{1}, X_{2}\right)$ where $\mathfrak{m}_{k}=\mathfrak{m}_{k}\left(X_{1}, X_{2}\right)$ is the span over $\mathbb{R}$ of the set

$$
\left\{\left[X_{j_{1}},\left[X_{j_{2}},\left[\cdots\left[X_{j_{k-1}}, X_{j_{k}}\right]\right] \cdots\right]\right]: j_{i}=1,2\right\} .
$$

For a smooth atlas of $M$ define a topology on $\mathscr{S}(M)$ by the metric

$$
\rho(v, w)=\sum_{k=0}^{\infty} \frac{1}{2^{k}} \frac{\|v-w\|_{k}}{1+\|v-w\|_{k}}, \quad v, w \in \mathscr{Y}(M),
$$

where $\|v-w\|_{k}$ is the supremum on $M$ of partial derivatives of order less than $k+1$ of components of $v-w$. Construct $\tilde{X}_{j}, \tilde{\mathfrak{m}}$, and $\tilde{\rho}(\cdot, \cdot)$ for a smooth manifold $\tilde{M}$ in the same way that $X_{j}, \mathfrak{m}$, and $\rho(\cdot, \cdot)$ were instead for $M$. Assume there is a Lie algebra epimorphism $\Phi: \mathfrak{m} \rightarrow \tilde{\mathfrak{m}}$.

LEMMA 2.1. The completion $\overline{\mathfrak{m}}$ of $\mathfrak{m}$ in the $\rho_{2}(\cdot, \cdot)=\rho(\cdot, \cdot)+\tilde{\rho}(\Phi(\cdot), \Phi(\cdot))$ topology is $\mathscr{Y}(M)$. 
Proof. Define

$$
\Delta_{\lambda \xi}=\sum_{k, l=0}^{\infty} \frac{\lambda^{k} \xi^{l}}{k ! l !} \operatorname{ad}^{k}\left(X_{1}\right)\left(\operatorname{ad}^{l}\left(X_{2}\right)\left(\left[X_{1}, X_{2}\right]\right)\right)
$$

which can be shown to converge for all $\lambda, \xi$ in the $\rho_{2}$ topology. Define

$$
V=\left\{\sum_{k=1}^{\infty} a_{k} \Delta_{\lambda_{k}} \xi_{k}: \sum_{k=1}^{\infty} a_{k}^{2}<\infty\right\} \subset \mathscr{S}(M) .
$$

Let $L$ be a nonzero element of $\mathscr{Y}(M)$ and $D$ a countable dense subset of $M$. Consider

$$
\begin{aligned}
v_{2 i-1} & =\left(L^{(1)}\left(p_{i}\right), \frac{1}{2} \Delta_{\lambda_{1} \xi_{1}}^{(1)}\left(p_{i}\right), \frac{1}{3} \Delta_{\lambda_{2} \xi_{2}}^{(1)}\left(p_{i}\right), \ldots\right) \in l_{2}, \\
v_{2 i} & =\left(L^{(2)}\left(p_{i}\right), \frac{1}{2} \Delta_{\lambda_{1} \xi_{1}}^{(2)}\left(p_{i}\right), \frac{1}{3} \Delta_{\lambda_{2} \xi_{2}}^{(2)}\left(p_{i}\right), \ldots\right) \in l_{2}, \quad p_{i} \in D,
\end{aligned}
$$

where for example $L^{(2)}\left(p_{i}\right)$ is the second component of $L\left(p_{i}\right)$ is a coordinate neighbourhood of $p_{i}$ and $l_{2}$ is the Hilbert space of elements $\left(a_{1}, a_{2}, \ldots\right)$ so that $\sum_{k=1}^{\infty} a_{k}^{2}<\infty$ with inner product $(\cdot, \cdot)$. Let $W$ be the span of the set of $v_{k}$. Since $\mathfrak{m}\left(X_{1}, X_{2}\right)$ is infinite dimensional there are $\lambda_{k}, \xi_{k}$ so that the map that maps $\left(a_{1}, a_{2}, a_{3}, \ldots\right)$ to $\left(a_{2}, a_{3}, \ldots\right)$ is injective on $W$. It will be injective on $\bar{W}$, the completion of $W$ in the $(\cdot, \cdot)$ topology. If, say, $L^{(1)} p_{k} \neq 0$ then $\left(0,1 / 2 \Delta_{\lambda_{1} \xi_{1}}^{(1)}\left(p_{k}\right), 1 / 3 \Delta_{\lambda_{2} \xi_{2}}^{(1)}\left(p_{k}\right), \ldots\right)$ is not an element of $\bar{W}$. There is then a nonzero $\left(a, a_{1}, a_{2}, \ldots\right) \in l_{2}$ with $a \neq 0$ so that

$$
a L\left(p_{i}\right)+a_{1} \Delta_{\lambda_{1} \xi_{1}}\left(p_{i}\right)+a_{2} \Delta_{\lambda_{2} \xi_{2}}\left(p_{i}\right)+\cdots=0, \quad \forall p_{i} \in D
$$

Since $D$ is dense this equation holds for all $p \in M$ hence $L \in V$.

THEOREM 2.2. If $m$ and $\tilde{m}$ are isomorphic then $M$ and $\tilde{M}$ are diffeomorphic.

Proof. Let $\Phi$ be an isomorphism of $\boldsymbol{m}$ and $\tilde{\mathfrak{m}}$. Let $\boldsymbol{m}$ have the topology $\rho_{2}$ and $\tilde{\mathfrak{m}}$ the topology $\tilde{\rho}_{2}(\cdot, \cdot)=\tilde{\rho}(\cdot, \cdot)+\rho\left(\Phi^{-1}(\cdot), \Phi^{-1}(\cdot)\right)$. The Lie bracket and $\Phi$ are continuous in this topology.

Let $A, B$ be the closure of open sets of $M$. By Lemma 2.1, there are vector fields $E, F \in \overline{\mathfrak{m}}$ with supports $A, B$, respectively. Let $\tilde{A}, \tilde{B}$ be the supports of $\Phi(E), \Phi(F)$, respectively. If $A \cap B \neq \varnothing$ then there are $E, F$ so that $[E, F] \neq 0$ hence $[\Phi(E), \Phi(F)] \neq 0$ consequently $\tilde{A} \cap \tilde{B} \neq \varnothing$ and vice versa.

Let $p_{0} \in M$ and let $A_{i}$ be the closure of open sets of $M$ and let $E_{i} \in \overline{\mathfrak{m}}$ have support $A_{i}$ and $\Phi\left(E_{i}\right)$ support $\tilde{A}_{i}$. It follows from the previous paragraph that if $A_{i+1} \subset A_{i}$ then $\tilde{A}_{i+1} \subset \tilde{A}_{i}$. The $A_{i}$ can be chosen so the diameters of $A_{i}, \tilde{A}_{i}$ approach zero and $\left\{p_{0}\right\}=\cap_{i=1}^{\infty} A_{i}$. Let $\left\{\tilde{p}_{0}\right\}=\cap_{i=1}^{\infty} \tilde{A}_{i}$. Let $\left\{B_{i}, \tilde{B}_{i}\right\}$ be another such sequence so that $\left\{p_{0}\right\}=$ $\cap_{i=1}^{\infty} B_{i}$ and suppose $\left\{\tilde{p}_{1}\right\}=\cap_{i=1}^{\infty} \tilde{B}_{i}$. Now the sequence of sets $\left\{A_{i} \cap B_{i}, \tilde{A}_{i} \cap \tilde{B}_{i}\right\}$ satisfy the same conditions so we must have that $\tilde{p}_{0}=\tilde{p}_{1}$. We thus have a well-defined map $\tau: M \rightarrow \tilde{M}$ so that $\tau\left(p_{0}\right)=\tilde{p}_{0}$ which on using the previous argument but with $\Phi^{-1}$ is a bijection.

Choose a coordinate neighborhood $(x, y)$ of $p_{0}$. Using $e^{\lambda \operatorname{ad}\left(X_{1}\right)}\left(X_{2}\right)$, let $\lambda(x, y)$ be the value of $\lambda$ required to move the integral curve of $X_{2}$ passing through $p_{0}$ along 
$X_{1}$ so that it intersects the point $(x, y)$. If $X_{1}\left(p_{0}\right)=0$ there is then a $Z_{1} \in \mathfrak{m}$ so that $X_{1}\left(p_{0}\right)+Z_{1}\left(p_{0}\right) \neq 0$. Now use Lemma 2.1 with $\Phi$ restricted to $\left\langle X_{1}+Z_{1}, X_{2}\right\rangle$. The map $\tau$ will remain unchanged. Similarly, using $e^{\xi \operatorname{ad}\left(X_{2}\right)}\left(X_{1}\right)$ we can construct $\xi(x, y)$.

Let $B$ be the closure of a neighborhood of $p_{0}$ then by Lemma 2.1 there is a sequence $Z_{00}^{(i)} \in V$ that converges to a smooth vector field with support $B$. Consider a coordinate system so that $\Delta_{\lambda \xi}(x, y)=\Delta_{\xi}(x+\lambda, y), p_{0}=(0,0)$ so $Z_{\lambda 0}^{(i)}$ constructed by replacing $\Delta_{\lambda_{k} \xi_{k}}$ by $\Delta_{\lambda_{k}+\lambda, \xi}$ in $Z_{00}^{(i)}$ will converge to a smooth vector field with support $B-(\lambda, 0)$ for sufficiently small $B$. Let $\Phi\left(Z_{00}^{(i)}\right)$ have support $\tilde{B}$. For a coordinate neighborhood $(\tilde{x}, \tilde{y})$ of $\tilde{p}_{0}=\tau\left(p_{0}\right)=(0,0)$ such that $\tilde{\Delta}_{\lambda \xi}(\tilde{x}, \tilde{y})=\tilde{\Delta}_{\xi}(\tilde{x}+\lambda, \tilde{y})$ we have $\Phi\left(Z_{\lambda 0}^{(i)}\right)$ will converge to a smooth vector field with support $\tilde{B}-(\lambda, 0)$. Using also a coordinate system so that $\Theta_{\lambda \xi}(x, y)=\Theta_{\lambda 0}(x, y+\xi)$ where $\Theta_{\lambda \xi}$ is constructed by interchanging $X_{1}$ and $X_{2}$ in $\Delta_{\lambda \xi}$ and on choosing smaller and smaller $B$ we can conclude that $\tau$ maps the point $(x, y)$ to $(\tilde{x}, \tilde{y})$ so that

$$
\lambda(x, y)=\tilde{\lambda}(\tilde{x}, \tilde{y}), \quad \xi(x, y)=\tilde{\xi}(\tilde{x}, \tilde{y}),
$$

where for example using $e^{\tilde{\lambda} \operatorname{ad}\left(\Phi\left(X_{1}\right)\right)}\left(\Phi\left(X_{2}\right)\right), \tilde{\lambda}(\tilde{x}, \tilde{y})$ is the value of $\tilde{\lambda}$ required to move the integral curve of $\Phi\left(X_{2}\right)$ passing through $\tilde{p}_{0}$ along $\Phi\left(X_{1}\right)$ until it intersects the point $(\tilde{x}, \tilde{y})$. Now $\lambda(x, y), \tilde{\lambda}(\tilde{x}, \tilde{y}), \xi(x, y), \tilde{\xi}(\tilde{x}, \tilde{y})$ are smooth functions so by taking derivatives of (2.8) it follows that $\tau$ is a diffeomorphism.

It follows from Theorem 2.2 that an automorphism of $m$ induces a diffeomorphism of $M$.

Define $V_{N}\left(X_{1}, X_{2}\right)=\sum_{k=1}^{N} \mathfrak{m}_{k}\left(X_{1}, X_{2}\right)$. The dimension of $V_{N}\left(X_{1}, X_{2}\right)$ is locally maximal if there is $\epsilon>0$ such that for analytic vector fields $Y_{j}$ on $M$ so that $\rho\left(X_{j}, Y_{j}\right)<\epsilon$ we have $\operatorname{dim} V_{N}\left(Y_{1}, Y_{2}\right) \leq \operatorname{dim} V_{N}\left(X_{1}, X_{2}\right)$.

Let $\left\{v_{q}, v_{q k}\right\}$ be a set of smooth vector fields on $M$ and let $\|\cdot\|$ be a norm on the span of this set. We also require that as $k \rightarrow \infty,\left\|v_{q k}-v_{q}\right\| \rightarrow 0$.

LEMMA 2.3. If $v_{1}, v_{2}, \ldots, v_{l}$ are linearly independent then there is an $N$ such that for $k>N, v_{1 k}, v_{2 k}, \ldots, v_{l k}$ are linearly independent.

Proof. Assume there are $t \rightarrow \infty$ so that for each $t$ we can find $a_{k t}$ so that

$$
a_{1 t} v_{1 t}+a_{2 t} v_{2 t}+\cdots+a_{l t} v_{l t}=0, \quad \sum_{k=1}^{l} a_{k t}^{2}=1 .
$$

There is a subsequence $\left\{a_{k s}\right\}$ and $a_{k}$ such that $\left|a_{k s}-a_{k}\right|<1 / s, k=1,2, \ldots, l$. Taking the limit as $s \rightarrow \infty$ we have $a_{1} v_{1}+a_{2} v_{2}+\cdots+a_{l} v_{l}=0$ with not all the $a_{k}$ zero which contradicts the linear independence of the set $\left\{v_{1}, v_{2}, \ldots, v_{l}\right\}$.

Let $\left\langle F_{1}, F_{2}\right\rangle$ be a free Lie algebra so $\operatorname{dim} V_{N}\left(X_{1}, X_{2}\right) \leq \operatorname{dim} V_{N}\left(F_{1}, F_{2}\right)<\infty$. Since the dimension of $V_{N}\left(X_{1}, X_{2}\right)$ is bounded for all analytic $X_{1}, X_{2}$ there are by Lemma 2.3 analytic $\hat{X}_{j}$ so that the dimension of $V_{N}\left(\hat{X}_{1}, \hat{X}_{2}\right)$ is locally maximal. In fact it can be shown that there is a Lie algebra $m\left(\hat{X}_{1}, \hat{X}_{2}\right)$ with analytic $\hat{X}_{j}$ so that the dimension of $V_{N}\left(\hat{X}_{1}, \hat{X}_{2}\right)$ is locally maximal for all $N$.

THEOREM 2.4. The Lie algebra $m\left(\hat{X}_{1}, \hat{X}_{2}\right)$ is a graded Lie algebra. 
Proof. Let $Y_{j}$ be the projection of $e^{-r^{2}}\left(\partial / \partial x_{p}\right), p=1,2, \ldots, m$ onto the tangent space at each point of $M$. Choose two of the projections say $Y_{1}, Y_{2}$ so that they span the tangent space at some point of $M$. For $\epsilon>0$ there is an embedding and coordinates on $M$ so that

$$
\left\|Y_{j}\left(x_{1}, x_{2}\right)-e^{-r^{2}} \frac{\partial}{\partial x_{j}}\right\|_{0}<\epsilon, \quad \forall\left(x_{1}^{2}+x_{2}^{2}\right) \leq 1, j=1,2
$$

Now by argument of Theorem 3.1 we have that $m\left(e^{-r^{2}}\left(\partial / \partial x_{1}\right), e^{-r^{2}}\left(\partial / \partial x_{2}\right)\right)$ is a graded Lie algebra. By Lemma 2.3 with a sufficiently small $\epsilon$ we have

$$
V_{N}\left(Y_{1}, Y_{2}\right)=\mathfrak{m}_{1}\left(Y_{1}, Y_{2}\right) \oplus \mathfrak{m}_{2}\left(Y_{1}, Y_{2}\right) \oplus \cdots \oplus \mathfrak{m}_{N}\left(Y_{1}, Y_{2}\right) .
$$

Define $E_{j}(t)=t Y_{j}+(1-t) \hat{X}_{j}$ and assume

$$
\mathscr{B}_{1}\left(E_{1}(t), E_{2}(t)\right)+\mathscr{B}_{2}\left(E_{1}(t), E_{2}(t)\right)+\cdots+\mathscr{B}_{N}\left(E_{1}(t), E_{2}(t)\right)=0
$$

with $\mathscr{B}_{p}\left(E_{1}(1), E_{2}(1)\right) \neq 0$ for some $p \leq N$ where $\mathscr{B}_{k}\left(E_{1}(t), E_{2}(t)\right)$ is

$$
a_{k 1}(t) B_{k 1}\left(E_{1}(t), E_{2}(t)\right)+\cdots+a_{k l_{k}}(t) B_{k l_{k}}\left(E_{1}(t), E_{2}(t)\right)
$$

and $B_{k q}\left(E_{1}(t), E_{2}(t)\right)$ is

$$
\left[E_{j_{1}}(t),\left[E_{j_{2}}(t),\left[\ldots,\left[E_{j_{k-1}}(t), E_{j_{k}}(t)\right]\right] \cdots\right]\right] \in \mathfrak{m}_{k}\left(E_{1}(t), E_{2}(t)\right),
$$

where the $j_{i}$ depend on $k$ and $q$. Since the dimension of $V_{N}\left(\hat{X}_{1}, \hat{X}_{2}\right)$ is locally maximal we have that there is a $t_{1}<1$ so that for $t \in\left(t_{1}, 1\right]$, (2.12) holds. The $a_{k q}(t)$ will then be polynomials in $t$. By (2.11) and Lemma 2.3 there is a $t_{0}>0$ so that for $t \in$ $\left[0, t_{0}\right)$ we must have $\mathscr{B}_{p}\left(E_{1}(t), E_{2}(t)\right)=0$. Now $\mathscr{B}_{p}\left(E_{1}(t), E_{2}(t)\right)$ is analytic in $t$ so $\mathscr{B}_{p}\left(E_{1}(1), E_{2}(1)\right)=0$ which is a contradiction hence

$$
V_{N}\left(\hat{X}_{1}, \hat{X}_{2}\right)=\mathfrak{m}_{1}\left(\hat{X}_{1}, \hat{X}_{2}\right) \oplus \mathfrak{m}_{2}\left(\hat{X}_{1}, \hat{X}_{2}\right) \oplus \cdots \oplus \mathfrak{m}_{N}\left(\hat{X}_{1}, \hat{X}_{2}\right) .
$$

THEOREM 2.5. The Lie algebras $\mathfrak{m}\left(X_{1}, X_{2}\right)$ and $\mathfrak{m}\left(\hat{X}_{1}, \hat{X}_{2}\right)$ are isomorphic.

Proof. Let $\epsilon>0$ be such that for all analytic $Y_{j}$ so that $\rho\left(Y_{j}, \hat{X}_{j}\right)<\epsilon$ we have that $\operatorname{dim} V_{N}\left(Y_{1}, Y_{2}\right)=\operatorname{dim} V_{N}\left(\hat{X}_{1}, \hat{X}_{2}\right)$. Let $Z_{j}-\hat{X}_{j} \in \oplus_{k=2}^{\infty} \mathfrak{m}_{k}\left(\hat{X}_{1}, \hat{X}_{2}\right)$ so that $\rho\left(Z_{j}, \hat{X}_{j}\right)<\epsilon$. Assume $\mathscr{B}_{B}\left(Z_{1}, Z_{2}\right)=0$ where

$$
\mathscr{B}\left(Z_{1}, Z_{2}\right)=\mathscr{B}_{1}\left(Z_{1}, Z_{2}\right)+\cdots+\mathscr{B}_{N}\left(Z_{1}, Z_{2}\right)
$$

and $\mathscr{B}_{k}\left(Z_{1}, Z_{2}\right)=a_{k 1} B_{k 1}\left(Z_{1}, Z_{2}\right)+\cdots+a_{k l_{k}} B_{k l_{k}}\left(Z_{1}, Z_{2}\right)$. The dimension of $V_{N}\left(Z_{1}, Z_{2}\right)$ will be locally maximal so by argument of Theorem 2.4 we have that $\mathscr{B}_{p}\left(Z_{1}, Z_{1}\right)=0$ for all $p \leq N$. Now $\mathscr{B}_{p}\left(Z_{1}, Z_{2}\right)=\mathscr{B}_{p}\left(\hat{X}_{1}, \hat{X}_{2}\right)+W_{p+1}=0$ where $W_{p+1}$ is an element of $\oplus_{k=p+1}^{\infty} \mathfrak{m}_{k}\left(\hat{X}_{1}, \hat{X}_{2}\right)$ hence $\mathscr{B}_{p}\left(\hat{X}_{1}, \hat{X}_{2}\right)=0$ for all $p \leq N$ consequently $\mathscr{B}\left(\hat{X}_{1}, \hat{X}_{2}\right)=0$. This holds for all such $\mathscr{B}$. Since the dimension of $V_{N}\left(\hat{X}_{1}, \hat{X}_{2}\right)$ is locally maximal we have $\mathscr{B}\left(Z_{1}, Z_{2}\right)=0$ if and only if $\mathscr{B}\left(\hat{X}_{1}, \hat{X}_{2}\right)=0$.

Define $E_{j}(t)=t X_{j}+(1-t) \hat{X}_{j}$. Using Lemma 2.3 there is a $t_{0}>0$ such that for $t \in\left[0, t_{0}\right), \rho\left(E_{j}(t), \hat{X}_{j}\right)<\epsilon$ and $B\left(E_{1}(t), E_{2}(t)\right)$ is a basis element of $V_{N}\left(E_{1}(t), E_{2}(t)\right)$ if 
and only if $B\left(\hat{X}_{1}, \hat{X}_{2}\right)$ is a basis element of $V_{N}\left(\hat{X}_{1}, \hat{X}_{2}\right)$. Using Lemma 2.1 with $\mathfrak{m}=\tilde{\mathfrak{m}}$ and $\Phi=$ id we can conclude that $\mathscr{B}\left(E_{1}(t), E_{2}(t)\right)=0$ for all $t_{0} \in\left[0, t_{0}\right)$. Now $\mathscr{B}\left(E_{1}(t), E_{2}(t)\right)$ is analytic in $t$ so when $t=1$ we have $\mathscr{B}\left(X_{1}, X_{2}\right)=0$. This holds for all $N$ and all such $\mathscr{B}$ so we can conclude there is an epimorphism

$$
\Phi: \mathfrak{m}\left(\hat{X}_{1}, \hat{X}_{2}\right) \longrightarrow \mathfrak{m}\left(X_{1}, X_{2}\right) .
$$

It can be shown that there are $W_{j} \in \overline{\mathrm{m}}$ so that $W_{j}, \Phi\left(W_{j}\right)$ are analytic and the dimension of $V_{N}\left(\Phi\left(W_{1}\right), \Phi\left(W_{2}\right)\right)$ is locally maximal for all $N$ and consequently $\Phi$ restricted to $\left\langle W_{1}, W_{2}\right\rangle$ is an isomorphism. By Lemma 2.1 the completion of $\left\langle W_{1}, W_{2}\right\rangle$ in the $\rho_{2}$ topology is $\mathscr{Y}(M)$ so $\Phi$ restricted to $m\left(\hat{X}_{1}, \hat{X}_{2}\right)$ is an isomorphism.

It follows from Theorems 2.4 and 2.5 that $\mathfrak{m}\left(X_{1}, X_{2}\right)$ is a graded Lie algebra.

By Lemma 2.3, Theorem 2.5, and the argument presented in the first paragraph of Theorem 2.4, it can be shown that if $B\left(X_{1}, X_{2}\right)$ is a basis element of $m\left(X_{1}, X_{2}\right)$ then the bracket $B\left(e^{-r^{2}}\left(\partial / \partial x_{1}\right), e^{-r^{2}}\left(\partial / \partial x_{2}\right)\right)$ is a basis element of $m\left(e^{-r^{2}}\left(\partial / \partial x_{1}\right), e^{-r^{2}}\left(\partial / \partial x_{2}\right)\right)$ and vice versa. It can also be shown that the dimension of $\mathfrak{m}_{k}\left(e^{-r^{2}}\left(\partial / \partial x_{1}\right), e^{-r^{2}}\left(\partial / \partial x_{2}\right)\right)$ grows polynomially with $k$ hence $\mathfrak{m}\left(X_{1}, X_{2}\right)$ is not a free Lie algebra.

THEOREM 2.6. The Lie algebras $\mathfrak{m}\left(X_{1}, X_{2}\right)$ and $\tilde{\mathfrak{m}}\left(\tilde{X}_{1}, \tilde{X}_{2}\right)$ are isomorphic if and only if $M$ and $\tilde{M}$ are diffeomorphic.

Proof. In Theorem 2.2 we showed that if $\mathfrak{m}\left(X_{1}, X_{2}\right)$ and $\tilde{\mathfrak{m}}\left(\tilde{X}_{1}, \tilde{X}_{2}\right)$ are isomorphic then $M$ and $\tilde{M}$ are diffeomorphic.

Let $\sigma$ be a diffeomorphism of $M$ and $\tilde{M}$. By the paragraph preceding this theorem we have that $B_{1}\left(X_{1}, X_{2}\right), \ldots, B_{l}\left(X_{1}, X_{2}\right)$ are basis elements of $V_{N}\left(X_{1}, X_{2}\right)$ if and only if $B_{1}\left(\tilde{X}_{1}, \tilde{X}_{2}\right), \ldots, B_{l}\left(\tilde{X}_{1}, \tilde{X}_{2}\right)$ are basis elements of $V_{N}\left(\tilde{X}_{1}, \tilde{X}_{2}\right)$. As in Theorem 2.5 we must have $\mathscr{B}\left(\sigma_{*}^{-1}\left(\tilde{X}_{1}\right), \sigma_{*}^{-1}\left(\tilde{X}_{2}\right)\right)=0$ hence $\mathscr{B}\left(\tilde{X}_{1}, \tilde{X}_{2}\right)=0$. This holds for all $N$ and $\mathscr{B}$ so we then have an epimorphism $\mathfrak{m}\left(X_{1}, X_{2}\right) \rightarrow \tilde{\mathfrak{m}}\left(\tilde{X}_{1}, \tilde{X}_{2}\right)$. Similarly, there is an epimorphism $\tilde{\mathfrak{m}}\left(\tilde{X}_{1}, \tilde{X}_{2}\right) \rightarrow \mathfrak{m}\left(X_{1}, X_{2}\right)$ so $\mathfrak{m}\left(X_{1}, X_{2}\right)$ and $\tilde{\mathfrak{m}}\left(\tilde{X}_{1}, \tilde{X}_{2}\right)$ are isomorphic.

As a possible application we can look at diffeomorphism classes of $S^{4}$. Let $P=$ $\left\{T_{i}\left(\theta_{1}, \theta_{2}, \theta_{3}, \theta_{4}\right): i=1, \ldots, 8\right\}$ be a set of polynomials in $\sin \theta_{j}, \cos \theta_{j}, j=1,2,3,4$ so that $P$ defines a homeomorphism of $S^{4}$ into a subset $S_{P}^{4}$ of $\mathbb{R}^{8}$. Let $\mathfrak{m}(P)$ be the Lie algebra with $k$ th grade $\mathfrak{m}_{k}(P)$ constructed by projecting $\partial / \partial x_{i}, i=1,2, \ldots, 8$ onto the tangent space of $S_{P}^{4}$ and choose four such projections so that they span the tangent space for some point of $S_{P}^{4}$. We can construct a finite dimensional graded Lie algebra

$$
\mathfrak{m}(P, N)=\frac{\mathfrak{m}(P)}{\bigoplus_{k=N}^{\infty} \mathfrak{m}_{k}(P)}
$$

for some positive integer $N$. If we can find two polynomial sets $P_{1}, P_{2}$, and an $N$ so that $\mathfrak{m}\left(P_{1}, N\right)$ and $\mathfrak{m}\left(P_{2}, N\right)$ are not isomorphic then we have at least two diffeomorphism classes of $S^{4}$.

3. Graded subalgebras constructed from atlases. Let $\left\{\left(U_{\alpha}, \phi_{\alpha}\right): \alpha \in I\right\}$ be an atlas of $M$ such that

(1) $U_{\alpha}$ is a neighborhood of $O_{\alpha}$ where the $O_{\alpha}$ are disjoint open connected subsets of $M$, the union of the closure of all the $O_{\alpha}$ is $M$. 
(2) $\phi_{\alpha}: U_{\alpha} \rightarrow \mathbb{R}^{n}$ are onto.

(3) $\phi_{\alpha} \circ \phi_{\beta}^{-1}$ are analytic.

(4) For $\sigma, \beta \in I$, a $\gamma \in I$ can be found such that $U_{\sigma} \cap U_{\gamma}=\varnothing$ and $U_{\beta} \cap U_{\gamma} \neq \varnothing$.

(5) For any set $\left\{\alpha_{1}, \alpha_{2}, \ldots, \alpha_{q}\right\}$ such that if $U=U_{\alpha_{1}} \cap U_{\alpha_{2}} \cap \cdots \cap U_{\alpha_{q}} \neq \varnothing$ then $U$ is connected and is a proper subset of $U_{\alpha_{1}} \cap \cdots \cap U_{\alpha_{i-1}} \cap U_{\alpha_{i+1}} \cap \cdots U_{\alpha_{q}}$ for all $i$.

Without loss of generality, in this section we consider manifolds so that $|I|<\infty$. Define

$$
\mathfrak{m}=\left\langle X_{\alpha p}: \alpha \in I, p \in \mathbb{N}=\{1,2, \ldots, n\}\right\rangle \subset \mathscr{P}(M)
$$

to be the Lie algebra generated by $X_{\alpha p}$ where $X_{\alpha p}$ is zero outside $U_{\alpha}$ and for points of $U_{\alpha}$ the push forward by $\phi_{\alpha}$ of $X_{\alpha p}$ is $e^{-r^{2}}\left(\partial / \partial x_{p}\right)$ so $\mathfrak{m}=\sum_{k=1}^{\infty} \mathfrak{m}_{k}$ where $\mathfrak{m}_{k}$ is the span over $\mathbb{R}$ of the set

$$
\left\{\left[X_{\beta_{1} j_{1}},\left[X_{\beta_{2} j_{2}},\left[\cdots\left[X_{\beta_{k-1} j_{k-1}}, X_{\beta_{k} j_{k}}\right]\right] \cdots\right]\right]: \beta_{i} \in I, j_{m} \in \mathbb{N}\right\} .
$$

If all the $\phi_{\alpha} \circ \phi_{\beta}^{-1}$ are rational functions it can be shown that the dimension of $\mathfrak{m}_{k}$ grows polynomially with $k$.

THEOREM 3.1. The Lie algebra $\mathrm{m}$ is a graded Lie algebra.

Proof. Consider for example an equation

$$
\begin{aligned}
& a_{1}\left[X_{\gamma_{1} j_{1}},\left[X_{\gamma_{2} j_{2}},\left[\cdots\left[X_{\gamma_{k-1} j_{k-1}}, X_{\gamma_{k} j_{k}}\right]\right] \cdots\right]\right] \\
& \quad+\cdots+a_{l}\left[X_{\sigma_{1} i_{1}},\left[X_{\sigma_{2} i_{2}},\left[\cdots\left[X_{\sigma_{m-1} i_{m-1}}, X_{\sigma_{m} i_{m}}\right]\right] \cdots\right]\right]=0 .
\end{aligned}
$$

We can write a component of (3.3) in the coordinates of $U_{\beta}$ as

$$
a_{1}\left[P_{11} F_{11}+\cdots+P_{1 \tau_{1}} F_{1 \tau_{1}}\right] e^{-q_{1} r^{2}}+\cdots+a_{l}\left[P_{l 1} F_{l 1}+\cdots+P_{l \tau_{l}} F_{l \tau_{l}}\right] e^{-q_{l} r^{2}}=0,
$$

where $P_{i j}\left(x_{1}, \ldots, x_{n}\right)$ is a polynomial and $F_{i j}\left(x_{1}, \ldots 0 c, x_{n}\right)$ is made of factors of partial derivatives of components of $X_{\alpha p}, \alpha \neq \beta$. $q_{i}$ is the number of times the factors of the form $X_{\beta p}$ appear in the $i$ th term of (3.3). Since the $X_{\alpha p}, \alpha \neq \beta$ are analytic for some point on the boundary of $U_{\beta}$ we must have $q_{i}=q_{j}$. Consequently writing equations like (3.3) in the coordinates of $U_{\gamma}$ for each $\gamma \in I$ and using condition 5 on the atlas allows us to conclude, $\mathfrak{m}=\oplus_{k=1}^{\infty} \mathfrak{m}_{k}$ and $\left[\mathfrak{m}_{i}, \mathfrak{m}_{j}\right] \subset \mathfrak{m}_{i+j}$ follows using properties of the Lie bracket.

Construct $\left\{\left(\tilde{U}_{\tilde{\alpha}}, \tilde{\phi}_{\tilde{\alpha}}\right): \tilde{\alpha} \in \tilde{I}\right\}, \tilde{X}_{\tilde{\alpha} p}, \tilde{\mathfrak{m}}, \tilde{\rho}(\cdot, \cdot)$ for a smooth manifold $\tilde{M}$ in the same way that $\left\{\left(U_{\alpha}, \phi_{\alpha}\right): \alpha \in I\right\}, X_{\alpha p}, \mathfrak{m}, \rho(\cdot, \cdot)$ were for $M$. Assume there is a Lie algebra isomorphism $\Phi: \mathfrak{m} \rightarrow \tilde{\mathfrak{m}}$. We can take $\Phi$ so that $\Phi\left(\mathfrak{m}_{1}\right)=\tilde{\mathfrak{m}}_{1}$. We now show this implies $M$ and $\tilde{M}$ are diffeomorphic.

LEMmA 3.2. There is a bijection $B: I \rightarrow \tilde{I}$ such that for all $p \in \mathbb{N}$, the support of $\Phi\left(X_{\alpha p}\right)$ is the closure of $\tilde{U}_{B(\alpha)}$.

Proof. Write $\Phi\left(X_{\alpha p}\right)=\tilde{Z}_{\tilde{\alpha}_{1}}+\cdots+\tilde{Z}_{\tilde{\alpha}_{m}}$ where $\tilde{Z}_{\tilde{\alpha}_{i}} \in \tilde{\mathfrak{m}}_{1}$ and $\operatorname{supp} \tilde{Z}_{\tilde{\alpha}_{i}}=\bar{U}_{\tilde{\alpha}_{i}}$. Assume $m>1$. There is a $\tilde{Z}_{\tilde{\alpha}_{i}}$, say $\tilde{Z}_{\tilde{\alpha}_{1}}$, so that $\operatorname{supp} \Phi^{-1}\left(\tilde{Z}_{\tilde{\alpha}_{1}}\right) \supset U_{\alpha}$. By condition 4 on the atlas there is a $\tilde{U}_{\tilde{\gamma}}$ such that $\tilde{U}_{\tilde{\alpha}_{1}} \cap \tilde{U}_{\tilde{\gamma}}=\varnothing$ and $\tilde{U}_{\tilde{\alpha}_{m}} \cap \tilde{U}_{\tilde{\gamma}} \neq \varnothing$. We can then find a $\tilde{X}_{\tilde{\gamma} q}$ so that $\left[\Phi\left(X_{\alpha p}\right),\left[\tilde{X}_{\tilde{\gamma} q}, \tilde{Z}_{\tilde{\alpha}_{m}}\right]\right] \neq 0$, hence $\Phi^{-1}\left(\left[\tilde{X}_{\tilde{\gamma} q}, \tilde{Z}_{\tilde{\alpha}_{m}}\right]\right)$ has support on 
$U_{\alpha}$. It can be checked that the bracket of an element of $m_{1}$ with an element of $\mathfrak{m}_{2}$ is not zero if they have common support. The intersection of $U_{\alpha}$ and the supports of $\Phi^{-1}\left(\tilde{Z}_{\tilde{\alpha}_{1}}\right)$ and $\Phi^{-1}\left(\left[\tilde{X}_{\tilde{\gamma} q}, \tilde{Z}_{\tilde{\alpha}_{m}}\right]\right)$ is then not empty so $\left[\Phi^{-1}\left(\tilde{Z}_{\tilde{\alpha}_{1}}\right), \Phi^{-1}\left(\left[\tilde{X}_{\tilde{\gamma} q}, \tilde{Z}_{\tilde{\alpha}_{m}}\right]\right)\right] \neq 0$ hence $\left[\tilde{Z}_{\tilde{\alpha}_{1}},\left[\tilde{X}_{\tilde{\gamma} q}, \tilde{Z}_{\tilde{\alpha}_{m}}\right]\right] \neq 0$ which is a contradiction since $\tilde{U}_{\tilde{\alpha}_{1}} \cap \tilde{U}_{\tilde{\gamma}}=\varnothing$ so $m=1$. Use the argument as just presented on $\Phi\left(X_{\alpha p}+X_{\alpha q}\right)$ to conclude that $\operatorname{supp} \Phi\left(X_{\alpha p}\right)=\overline{\tilde{U}}_{\tilde{\alpha}_{1}}$, for each $p \in \mathbb{N}$. Define $B: I \rightarrow \tilde{I}$ by $B(\alpha)=\tilde{\alpha}_{1}$. If $\operatorname{supp} \Phi\left(X_{\beta_{1} p_{1}}\right)=\operatorname{supp} \Phi\left(X_{\beta_{2} p_{2}}\right)$ then by letting $\tilde{Z}_{B\left(\beta_{1}\right)}=\Phi\left(X_{\beta_{1} p_{1}}+X_{\beta_{2} p_{2}}\right)$ and using a similar argument as just presented on $\Phi^{-1}\left(\tilde{Z}_{B\left(\beta_{1}\right)}\right)$ allows us to conclude that $B$ is bijective.

THEOREM 3.3. If $\mathfrak{m}$ and $\tilde{\mathfrak{m}}$ are isomorphic then $M$ and $\tilde{M}$ are diffeomorphic.

Proof. We can write $L \in \mathscr{Y}(M)$ as $L=\sum_{\alpha \in I} L_{\alpha}$ where $L_{\alpha} \in \mathscr{S}(M)$ has support, a compact subset of $U_{\alpha}$. By Lemmas 3.2 and 2.1 we have that $L_{\alpha}$ is in the completion of $\left\langle X_{\alpha p}: p \in \mathbb{N}\right\rangle$ in the $\rho_{2}$ topology. Now use the argument of Theorem 2.2.

We now look at how the graded Lie algebras constructed from different atlases satisfying the five conditions differ. Let $\left\{\left(U_{\alpha}, \phi_{\alpha}\right),\left(\hat{U}_{\beta}, \hat{\phi}_{\beta}\right): \alpha \in I \backslash\{\beta\}\right\}$ be an atlas of $M$ satisfying the five conditions on an atlas and require also that $U_{\beta} \cap U_{\gamma} \neq \varnothing$ if and only if $\hat{U}_{\beta} \cap U_{\gamma} \neq \varnothing$ for all $\gamma \in I$. Define $\hat{X}_{\beta p}$ to be the push forward of $X_{\beta p}$ by $\hat{\phi}_{\beta}^{-1} \circ \phi_{\beta}$. Define

$$
\hat{\mathfrak{m}}=\left\langle X_{\alpha p}, \hat{X}_{\beta p}: \alpha \in I \backslash\{\beta\}, p \in \mathbb{N}\right\rangle
$$

with $k$ th grade $\hat{\mathfrak{m}}_{k}$.

We can in fact choose $\hat{U}_{\beta}$ and $\hat{\phi}_{\beta}$ so that the dimension of $\sum_{k=1}^{N} \hat{\mathfrak{m}}_{k}$ is locally maximum for all $N$.

THEOREM 3.4. There is an epimorphism $\hat{\mathfrak{m}} \rightarrow \mathfrak{m}$ mapping $\hat{X}_{\beta p}$ to $X_{\beta p}$ and $X_{\alpha p}$ to $X_{\alpha p}$ for $\alpha \neq \beta$.

Proof. Without loss of generality, we can let $n=1$ and define $X_{\alpha}=X_{\alpha 1}$. Define a diffeomorphism

$$
\theta^{-1}:(-1,1) \rightarrow \mathbb{R}, \quad x \longmapsto \frac{x}{1-x^{2}}
$$

Write $\hat{\phi}_{\beta}^{-1} \circ \phi_{\beta}$ in the $\theta \circ \phi_{\beta}$ coordinates of $U_{\beta}$ as $x \mapsto \hat{x}=x+T(x)$ and $x=\hat{x}+S(\hat{x})$. Let

$$
T^{(N)}(x)=e^{-1 /(1+\delta)^{2}-x^{2}} \sum_{l=0}^{N} b_{l}^{(N)} x^{l}, \quad \delta>0
$$

be such that as $N \rightarrow \infty, T^{(N)}$ converges uniformly to $T$ on $[-1,1]$. Define $\hat{X}_{\beta}^{(N)}$ and $\hat{\mathfrak{m}}^{(N)}$ by replacing $T(x)$ by $T^{(N)}(x)$ in the definition of $\hat{X}_{\beta}$ and $\hat{\mathfrak{m}}$. Consider for example an equation of the form

$$
\begin{aligned}
a_{1}^{(N)}\left[\hat{X}_{\beta}^{(N)},\left[X_{\gamma_{2}},\left[\cdots\left[X_{\gamma_{k-1}}, \hat{X}_{\beta}^{(N)}\right]\right] \cdots\right]\right] \\
\quad+\cdots+a_{l}^{(N)}\left[X_{v_{1}},\left[\hat{X}_{\beta}^{(N)},\left[\cdots\left[\hat{X}_{\beta}^{(N)}, X_{v_{k}}\right]\right] \cdots\right]\right]=0 .
\end{aligned}
$$

By the argument of Theorem 3.1, each term will contain the same number of factors of the form $\hat{X}_{\beta}^{(N)}$. We can write $X_{\beta} \mapsto \hat{X}_{\beta}$ in the $\theta \circ \phi_{\beta}$ coordinates of $U_{\beta}$ as

$$
e^{-\left[x /\left(1-x^{2}\right)\right]^{2}} \frac{\left(1-x^{2}\right)^{2}}{1+x^{2}} \frac{d}{d x} \longmapsto \frac{e^{-\left[(x+S(x)) /\left(1-(x+S(x))^{2}\right)\right]^{2}}}{1+S^{\prime}(x)} \frac{\left(1-(x+S(x))^{2}\right)^{2}}{1+(x+S(x))^{2}} \frac{d}{d x} .
$$


With this in mind, we can write (3.8) in the $\theta \circ \phi_{\beta}$ coordinates of $U_{\beta}$ after clearing denominators and exponentials as

$$
\begin{aligned}
a_{1}^{(N)} & {\left[\left(P_{11}^{(N)}+Q_{11}^{(N)}\right) F_{11}^{(N)}+\cdots+\left(P_{1 \tau_{1}}^{(N)}+Q_{1 \tau_{1}}^{(N)}\right) F_{1 \tau_{1}}^{(N)}\right]+\cdots } \\
& +a_{l}^{(N)}\left[\left(P_{l 1}^{(N)}+Q_{l 1}^{(N)}\right) F_{l 1}^{(N)}+\cdots+\left(P_{l \tau_{l}}^{(N)}+Q_{l \tau_{l}}^{(N)}\right) F_{l \tau_{l}}^{(N)}\right]=0
\end{aligned}
$$

where the $F_{i j}^{(N)}(x)$ are made of factors of derivatives of the component of $X_{\alpha}, \alpha \neq \beta$. $P_{i j}^{(N)}(x)$ is a polynomial and $Q_{i j}^{(N)}$ is a polynomial in $x, S^{(N)}$, and derivatives of $S^{(N)}$. Each term of $Q_{i j}^{(N)}$ contains a $S^{(N)}$ or a derivative of $S^{(N)}$ as a factor. Since $S^{(N)}$ is analytic on $(-1-\delta, 1+\delta),(3.10)$ holds for all points of $(-1-\delta, 1+\delta)$. On the boundary of $(-1-\delta, 1+\delta), S^{(N)}$ and all its derivatives are zero hence for all points of $(-1,1)$

$$
a_{1}^{(N)}\left[P_{11}^{(N)} F_{11}^{(N)}+\cdots+P_{1 \tau_{1}}^{(N)} F_{1 \tau_{1}}^{(N)}\right]+\cdots+a_{l}^{(N)}\left[P_{l 1}^{(N)} F_{l 1}^{(N)}+\cdots+P_{l \tau_{l}}^{(N)} F_{l \tau_{l}}^{(N)}\right]=0
$$

Equation (3.8) then holds when $\hat{X}_{\beta}^{(N)}$ is replaced by $X_{\beta}$. There is then an epimorphism $\hat{\mathfrak{m}}^{(N)} \rightarrow \mathfrak{m}$ taking $\hat{X}_{\beta}^{(N)}$ to $X_{\beta}$ and $X_{\alpha}$ to $X_{\alpha}$ for $\alpha \neq \beta$. Now let $N \rightarrow \infty$ and use the local maximality of the dimension of $\sum_{k=1}^{N} \hat{\mathfrak{m}}_{k}$ for all $N$.

Let $\hat{\imath}=\left\langle\hat{L}_{\alpha p}: \alpha \in I, p \in \mathbb{N}\right\rangle$ be a graded Lie algebra with $k$ th grade $\hat{\mathfrak{l}}_{k}$ constructed as was $\mathfrak{m}$ and such that the dimension of $\sum_{k=1}^{N} \hat{\mathfrak{l}}_{k}$ is locally maximum for all $N$. We can use Theorem 3.4 to show that there is an $\epsilon>0$ such that for all such $\hat{\Gamma}$ with $\rho\left(\hat{L}_{\alpha p}, X_{\alpha p}\right)<\epsilon$ for all $\alpha \in I, p \in \mathbb{N}$ we have an epimorphism $\hat{\imath} \rightarrow m$.

\section{REFERENCES}

[1] H. Whitney, Differentiable manifolds, Ann. of Math. II. Ser. 37 (1936), 645-680. Zbl 015.32001.

KARL De PAEPE: Box 1174, VIRDEN MB RoM 2CO, CANADA

E-mail address: k.depaepe@utoronto.ca 


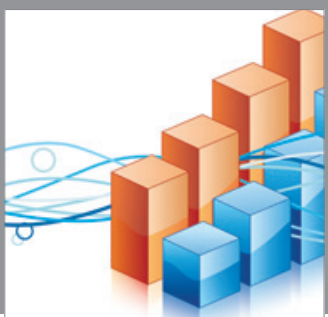

Advances in

Operations Research

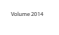

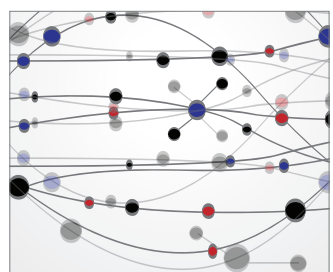

\section{The Scientific} World Journal
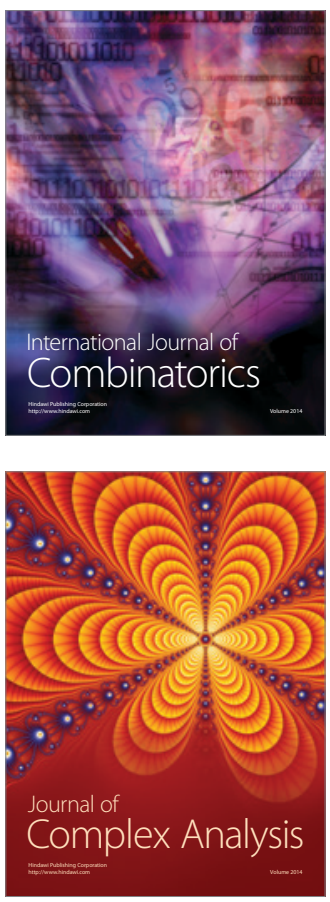

International Journal of

Mathematics and

Mathematical

Sciences
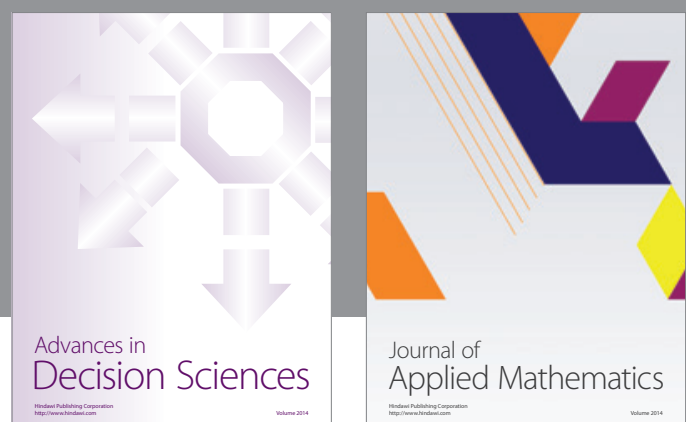

Journal of

Applied Mathematics
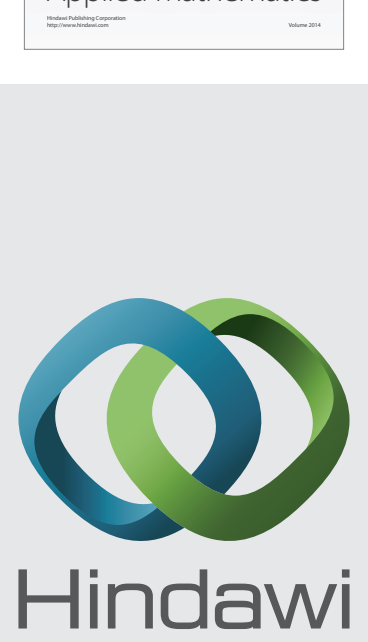

Submit your manuscripts at http://www.hindawi.com
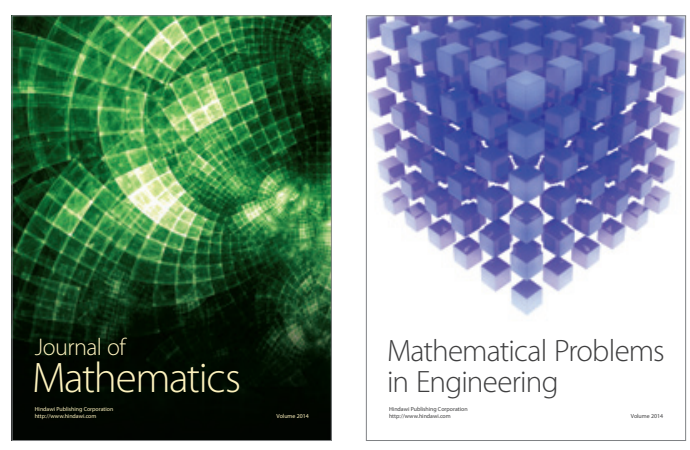

Mathematical Problems in Engineering
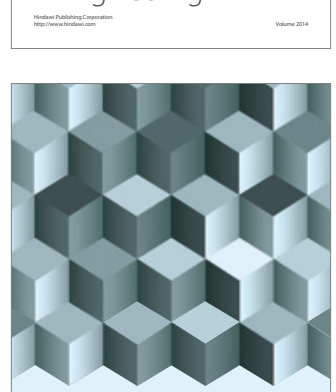

Journal of

Function Spaces
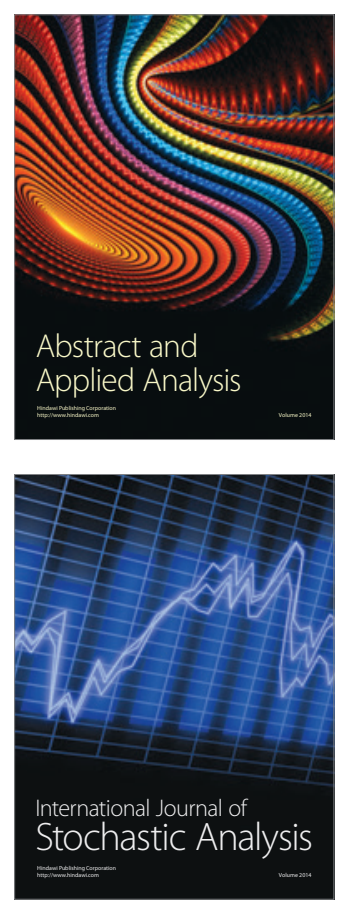

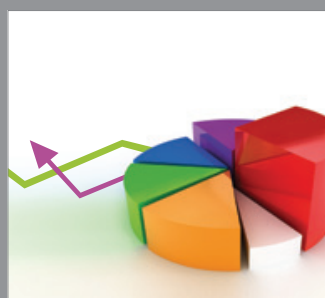

ournal of

Probability and Statistics

Promensencen
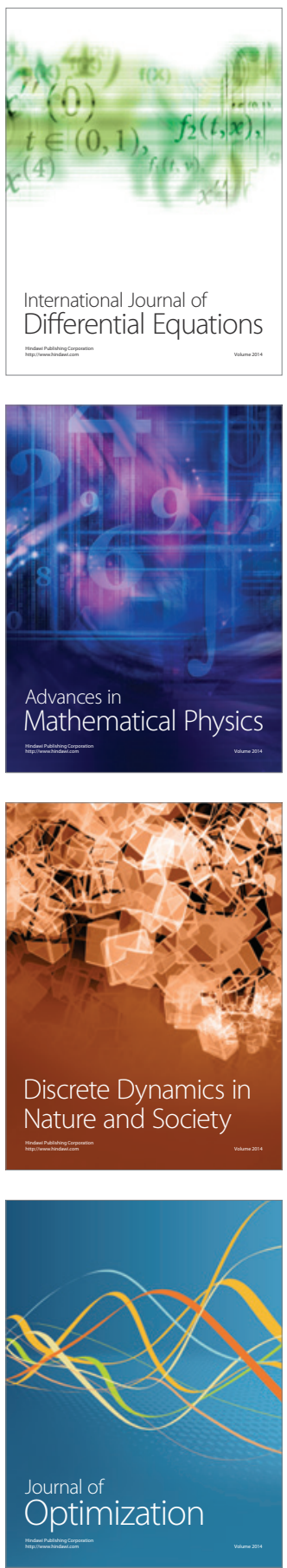